\title{
FGMP: assessing fungal genome completeness
}

\author{
Ousmane H. Cissé 1, $^{*}$ (iD and Jason E. Stajich ${ }^{1^{*}}$
}

\begin{abstract}
Background: Inexpensive high-throughput DNA sequencing has democratized access to genetic information for most organisms so that research utilizing a genome or transcriptome of an organism is not limited to model systems. However, the quality of the assemblies of sampled genomes can vary greatly which hampers utility for comparisons and meaningful interpretation. The uncertainty of the completeness of a given genome sequence can limit feasibility of asserting patterns of high rates of gene loss reported in many lineages.

Results: We propose a computational framework and sequence resource for assessing completeness of fungal genomes called FGMP (Fungal Genome Mapping Project). Our approach is based on evolutionary conserved sets of proteins and DNA elements and is applicable to various types of genomic data. We present a comparison of FGMP and state-of-the-art methods for genome completeness assessment utilizing 246 genome assemblies of fungi. We discuss genome assembly improvements/degradations in 57 cases where assemblies have been updated, as recorded by NCBI assembly archive.

Conclusion: FGMP is an accurate tool for quantifying level of completion from fungal genomic data. It is particularly useful for non-model organisms without reference genomes and can be used directly on unassembled reads, which can help reducing genome sequencing costs.
\end{abstract}

Keywords: Assembly, Conserved elements, Gene model

\section{Background}

The recent explosion of high-throughput sequencing methods and analytic tools has made sequencing easier and cheaper for nearly all species across the tree of life including uncultivable organisms. However, the quality and completeness of these genomes can vary due to challenges in assembling repeat rich regions and variable or insufficient sequencing coverage [1]. Large-scale sequencing projects such as the microbial dark matter project [2], the Human Microbiome Project [3] or the 1000 fungal genomes project (http://1000.fungalgenomes.org) have produced thousands of microbial genome assemblies. The rapid generation and release of draft data is contributing important and useful datasets that are extensively used for studies of pathology, evolution, and discovery of enzymes or pathways. Variable quality and completeness of draft genomes can impact the inferences drawn regarding

\footnotetext{
*Correspondence: ousmanecis@gmail.com; jason.stajich@ucr.edu ${ }^{1}$ Department of Microbiology \& Plant Pathology and Institute for Integrative Genome Biology, University of California-Riverside, Riverside, CA 92521, USA Full list of author information is available at the end of the article
}

gene content, transposable element load, and genome size. There is a need to quantify a genome's completeness to provide context of the quality of information that can be inferred from it. This work is also motivated by observations that lineage specific gene loss is an important driving force in evolution, especially in fungi $[4,5]$, and the accuracy of conclusions drawn about the patterns of missing genes requires comparisons among similar quality genomes.

Approaches to assess the quality and completeness of a genome have been proposed using nearly 100 different metrics [6]. Unfortunately, most of these metrics are generally not applicable to non-model species because they require a substantial amount of additional high-quality data (e.g. fosmids, reference genomes, optical maps) that can be expensive or infeasible to obtain for a large number of samples. Currently, few methods attempt to estimate the amount of missing data in an assembly without prior knowledge. One of the most popular approaches, CEGMA estimates the completeness to the presence of set of 248 single copy gene markers $[7,8]$. Although CEGMA has been used in numerous studies, a key issue is that markers were 
selected from only six model eukaryotic species and the ubiquity and detections of these markers may not be consistent as more distant lineages are sampled. CEGMA has been recently discontinued and the authors recommend using alternative tools (http://www.acgt.me/blog/2015/5/ 18/goodbye-cegma-hello-busco). The concept has been recently revisited and updated with clade-focused sets of protein coding gene markers in BUSCO [9]. Another set of 246 single copy fungal gene families has been proposed by FUNYBASE [10]. The latter provides a set of conserved fungal genes but the tools are not explicitly developed to assess genome completeness. Furthermore, the FUNYBASE database was generated in 2010 while a broader sampling of diverse fungal genomes is now available [11].

To build a dataset of independent markers to assess completeness, typically, single copy orthologous genes are chosen. Multi-copy gene families are systematically filtered out in these selections, but their utility, as well as that of alternative, non-protein coding gene markers has not been fully explored in assessing genome completeness. Two summary statistics of genome assemblies are frequently used to evaluate quality and completeness. The N50 and L50 statistics [12] which describe the level of fragmentation of the assembly are computed based on the lengths of assembly scaffolds or contigs. Both statistics utilize a sorted list of largest to smallest sizes of contigs, where L50 is the length (in bases) of the shortest contig for which $50 \%$ of the genome can be contained within contigs of that size or larger, and N50 is the number of contigs that when summed their length is half of the assembly size [13]. Note that unfortunately these two concepts are swapped in some tools, where N50 means length and L50 means the count. Still other methods measure the number error per bases or assembly inconsistencies to predict genome quality $[14,15]$.

In the present study, we focused on the fungal kingdom. Fungal genome sizes vary from several megabases $(\mathrm{Mb})$ to nearly $1000 \mathrm{Mb}$ [11]. A primary motivation of this work is to provide a realistic estimation of assembly completeness for fungal genomes. The precision depends on the ability to accurately identify genes, which can appear artifactually fragmented by an incomplete assembly or appear lost due to more rapidly evolving loci in some lineages. The nature, evolutionary trajectory and loss likelihood of genes need to be considered when calculating genome completeness from gene content. We propose a novel set of markers and build a pipeline to assess their presence in genome assemblies called FGMP (Fungal Genome Mapping Project). Our multistep approach extends previous approaches by integrating identifiable fungal proteins and highly conserved non-coding regions. The protein markers selected include both single and multi-copy markers and have only a 50\% overlap with previously published datasets providing a different dimension of sequence evolution to evaluate the completeness. Highly conserved non-coding regions of fungal genomes are novel resources we have developed and incorporated into assessment of genome completeness in FGMP. Lastly, we use a multisampling approach coupled to a rarefaction analysis to search for markers in unassembled sequencing reads, which bypass the need for an assembly. Therefore, using FGMP, a researcher can quickly assess the quality of a set of reads in hand before attempting an assembly, which can be computationally expensive. Finally, we described a side-by-side comparison of our tool with state-of arts methods over 246 fungal species with genome assemblies of varying ranges of quality. We captured assembly improvements/degradations in 57 fungal species with more than one released assembly, as recorded in NCBI assembly archive. The modular construction of this work can be a valuable tool for genome completion estimation that can be easily incorporated in more complex pipeline.

\section{Implementation}

A typical run of FGMP consists of three steps. First, a set of raw gene models (proteins) is generated from the queried assembly which are further filtered down to high confidence genes in subsequent steps. Second, the presence of highly conserved non-coding fungal DNA elements (> 200 nucleotides) is estimated. Third, the copy number of ubiquitous multi-copy protein families is determined to track possible mis-assemblies or collapsed duplicated regions. The FGMP workflow is diagrammed in Fig. 1 and methodology further detailed in the following sections. FGMP is not intended to assess the completeness of metagenome assemblies. Input data are expected to belong to a single species, and bacterial contaminants should be removed beforehand.

\section{Reference data preparation}

FGMP is primarily designed for assessment of fungal genome quality using defined sets of conserved proteins, noncoding highly conserved DNA elements (HDE) and multi-copy protein families. All the datasets are included in FGMP package and a stable released version is available at DOI: https://doi.org/10.5281/zenodo.1453438. Installation is available via Bioconda package system as "conda install -c bioconda fgmp" [16]. Alternatively, a step by step manual installation guide is provided at https://github.com/stajichlab/FGMP.

To generate the protein markers, we analyzed a phylogenomic dataset of 25 fungi with complete genomes covering major fungal lineages (Additional file 1). In total, 164,232 proteins were analyzed. Our goal is to capture the fungal protein diversity rather than focusing on universally conserved proteins. We focused on obtaining a set of diverse proteins to be used for initial identification of candidate regions and training sets for gene predictions. Our 


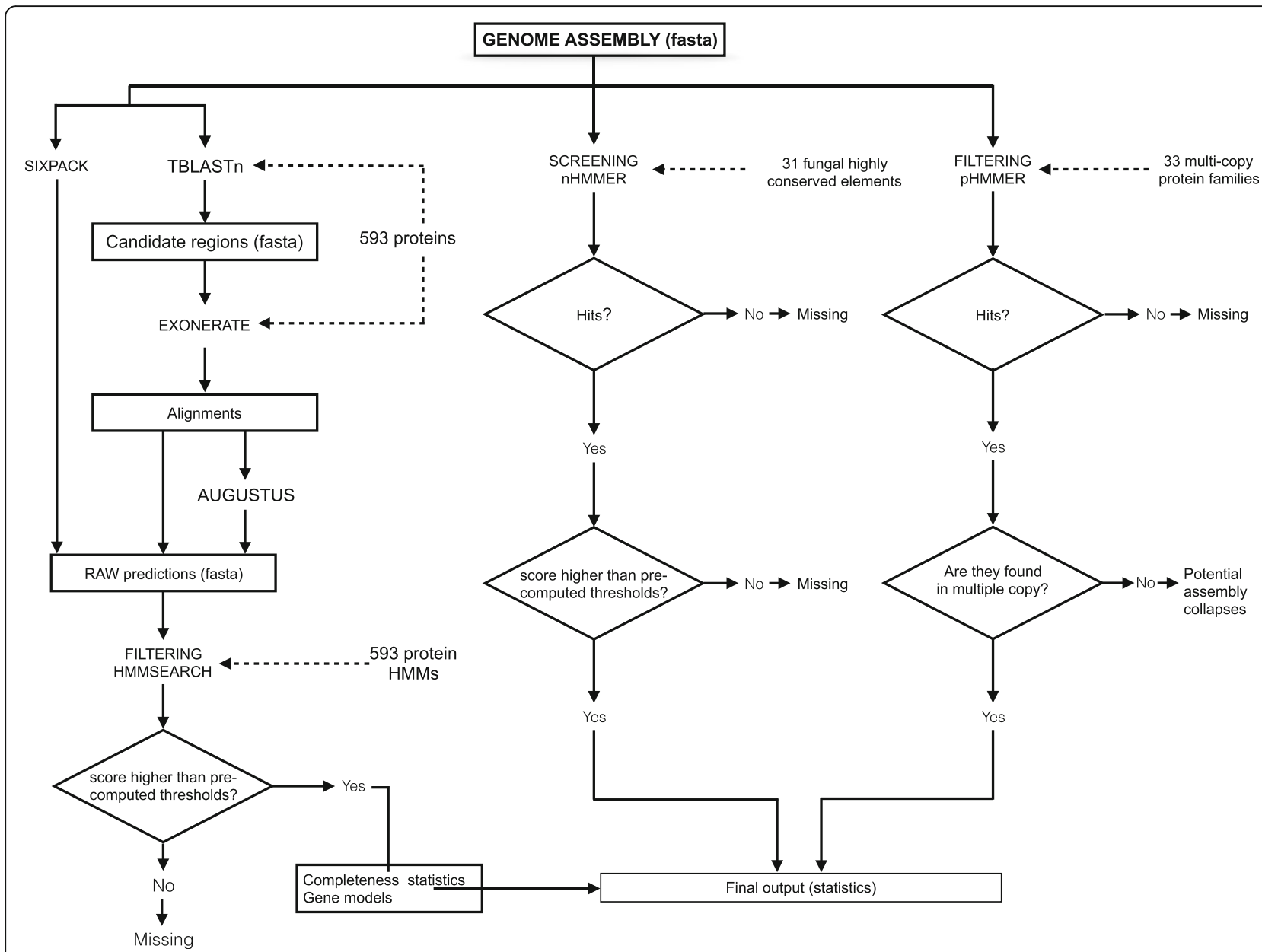

Fig. 1 The FGMP workflow. A typical workflow consists of three sequential modules (indicated by the boxes). The first module (FGMP_PROT) automates the use of different programs to evaluate the genome completeness based on pre-defined protein and nucleotide markers. Additional modules evaluate the patterns of conservation fungal multi-copy protein families. FGMP protein and nucleotide datasets are derived from 25 and nine fungal species, respectively (indicated as dotted arrows)

assumption is that irrespective to the phylogenetic classification of species under analysis, our diversified set of proteins would contain a homolog with sufficient protein similarity to generate a valid gene model. With these concepts in mind, we identified orthologous protein families using OMA [17] followed by inspection of the ortholog clusters using BLAST [18] and full-length pairwise alignments generated using needle from EMBOSS package [19]. We extracted 7773 protein families present in at least four species and use them to construct Hidden Markov Models (HMMs) using HMMER3 [20]. Protein families here refer to orthologous groups. In parallel, we selected a single most informative protein in each of these families using M-COFFEE [21], which corresponds to the sequence containing information that is lacking in other sequences of a multiple sequence alignment. The sequences lacking information in the other sequences might be due be mispredictions and/or protein domain loss, which are common in most annotated genomes. We computed the significance scores as follows: each protein of each cluster was compared to its corresponding HMM and the threshold corresponds to $80 \%$ of the score of the protein presenting lowest the score. The use of the full set of 7773 proteins appeared to be excessively demanding in terms of computational resources, even for small sized genome assemblies (e.g. < 8 megabases; data not shown; the 7773 protein markers are available at DOI: https://doi.org/10. 5281/zenodo.2541782). To reduce the computational burden, we then filtered out potentially paralogous sequences using PHMMER with an E-value of $10^{-50}$ as cut off [20], and applied the following rules: (i) a marker should be present in at least $99 \%$ of the species and (ii) should be unambiguously identifiable based on the alignment score of the protein against the HMM of the family. This filtering reduces our markers dataset to 593 proteins of which $60.3 \%$ are from single copy genes contrasting other published strategies, which exclude multi-copy gene families (BUSCO and CEGMA). These 593 representative protein 
sequences are aligned to the queried genome assembly to identify genomic regions that encode homologous genes using tBLASTn [18]. Once candidate regions are narrowed down by these translated alignment searches, fine-grain alignments of the proteins to these homologous regions in the target genome are generated using splice-site aware protein2genome alignment with EXONERATE [22]. These alignments-based gene models are used as training sets for AUGUSTUS [23]. The predicted proteins including both AUGUSTUS gene models and translated EXONERATE alignment matches are then searched against $593 \mathrm{HMMs}$ to identify the originating genes. FGMP assigns confidence in these predictions based on pre-defined thresholds. We benchmarked FGMP using the full (7773) and reduced set of proteins (593) on our subsequent analyses and found no significant differences in completeness estimates between the two sets of proteins. Protein domains were inferred using InterProScan [24] and mapped to gene ontology terms with InterPro2GO (http:// www.geneontology.org/external2go/interpro2go).

To identify highly conserved non protein-coding fungal DNA elements, we performed pairwise whole genome alignments of nine fungi using LAST [25]. The phylogeny of the selected species is presented in the Additional file 2. Coding regions were removed from alignments based on NCBI annotations using BEDtools [26]. The filtering was carried out using a computational pipeline combining enriched motifs and alignments from MEME [27], BLASTn [18] and EMBOSS 'needle' [19]. A total of 31 non-coding highly conserved regions in each species were extracted with a requirement that loci be at least 200 nucleotides long with a minimum of $70 \%$ global identity. These alignments were converted into HMMs using NHMMER from HMMER version 3.1b2. Comparison of these non-coding segments to Rfam database v14.0 [28] detects only one putative non coding RNA (FGMP.Ribo_CE.scaffold_35.127.484) with similarity to the Intron gpI family (RF00028), which suggests that most of the conserved DNA elements are true non-coding regions. To investigate whether our conserved DNA elements are specific to fungi, we aligned FGMP DNA markers to a custom non-fungi eukaryotic genomic database using nhmmer with an e-value of $10^{-20}$ as cut off. The database includes Toxoplasma gondii (NCBI accession number GCF_000006565.2), Trichomonas vaginalis (GCF_000002825.2), Leishmania major (GCF_000002725.2); Leishmania donovani (GCF_000227135.1), Plasmodium vivax (GCF_000002415.2), Plasmodium falciparum (GCF 000002415.2), Plasmodium yoelii (GCF_900002385.1). Seventeen out of 33 elements had significant hits against the database, although no full-length match was detected. This finding suggests that these half of FGMP conserved DNA elements could be present in other eukaryotes.
To identify ubiquitous multi-copy protein families within our set of 593 proteins, we surveyed 345 fungal genomes. Thirty-three protein families appear to have more than one copy in all the genomes (HMMSEARCH, E-value $<10^{-50}$ ). The dataset includes 15 helicases, six kinases, six chaperonin and six diverse proteins. For each of these 33 proteins, we consider the minimum number of copies. FGMP records the number of copies of these 33 proteins and reports when the copy number is lower than expected. The current implementation cannot differentiate independent copies from unassembled data.

Lastly, FGMP can estimate the level of completion directly from raw sequences using an iterative reservoir sampling approach. The process starts by splitting the set of reads by blocks of $10^{4}$ sequences. Then, FGMP randomly selects 1000 blocks using a reservoir sampling approach. This parameter can be modified by the user. Chunks of sequences are iteratively screened for presence of 593 protein makers using BLASTx [24]. The number of markers detected is recorded at each iteration. FGMP will stop after 20 successive unsuccessful attempts to detect new markers.

\section{Results}

\section{Protein markers comparison}

To determine if there is overlap among protein markers, we compared FGMP protein markers to proteins used by other tools. Noting that CEGMA has been recently discontinued and FUNYBASE is outdated, this comparison is only for an historical perspective. A total of 7773 FGMP markers were originally obtained, which was reduced to 593 after the removal of ambiguous markers (see Reference data preparation). We compared the markers selected for FGMP (593 proteins) to those used in CEGMA (248 families, 1488 proteins), BUSCO fungi (1438 proteins) and FUNYBASE (246 families, 5166 proteins). Using reciprocal best BLASTp (E-value $<10^{-5}$ ), $49.5 \%$ of FGMP protein markers are not found in the other datasets whereas the proportions of unique markers using the same criterion in CEGMA, FUNYBASE and BUSCO are respectively 21.7, 10.5 and $69.8 \%$ (see Additional file 3). FGMP proteins tend to be conserved in other eukaryotes but their utility outside the fungal kingdom is not explored in the present study. Transferases and transporters are common (13\%). Kinases and helicases are overrepresented in FGMP protein dataset where they represent 10 and $5 \%$ of 593 protein markers, respectively as compared to 0.8 and $2 \%$ of CEGMA makers; 3.3 and $2 \%$ in FUNYBASE markers; 3.3 and $0.7 \%$ of BUSCO fungi markers. Kinases and helicases are multi-copy protein families in nearly all fungi and likely this multicopy property is why these genes are not present in other datasets, which actively restrict gene 
duplicates. Most FGMP transferases have homologs in bacteria and archaea, suggesting that they are ancient. Most of the helicases present similarities with archaeal or bacterial proteins as well (PHMMER e-value of $10^{-5}$ ), and might represent a mix of ancient and derived forms [29].

\section{Comparison with related tools}

To investigate the ability of different software to detect changes in genome assemblies' quality, we analyzed the initial and subsequently updated genome assemblies of 45 fungi. As FGMP measures the genome completeness using protein and highly conserved non-coding elements, we refer to these modules as FGMP_PROT and FGMP_HCE, respectively. This is because FGMP_PROT is roughly equivalent to BUSCO or CEGMA, but FGMP_HCE is unique to FGMP. FGMP_PROT and FGMP_HCE predict increases in genome completeness in 35 and $31 \%$ of the 45 species, respectively whereas BUSCO-fungi predicts increases in 53\% and CEGMA in $60 \%$ of the species (Fig. 2 panels a to d). Overall, the compared methods agree on 16 out of 45 species (Fig. 2 panel e). BUSCO and CEGMA genome completeness predictions agree on 31 out of 45 genomes, whereas BUSCO and FGMP_PROT/FGMP_HCE predictions agree on 26 and 21 genomes, respectively. CEGMA and FGMP_PROT/FGMP_HCE agree on 28 and 25 genomes, respectively. FGMP_PROT and FGMP_HCE predictions agree on 24 genomes. FGMP is the most conservative method at assigning increase in completeness between versions and CEGMA is the most permissive. No statistically significant correlation between FGMP and BUSCO results and various genome statistics was observed (i.e. N50, sequencing coverage, or the sequencing technology used), which is consistent with Assemblathon results [6] showing that completeness metrics are not necessarily correlated among genome assembly statistics. However, CEGMA results appeared to be correlated to the sequencing technology used (Spearman rho $=0.2$ ), that is, assemblies generated exclusively with short reads (e.g. Illumina) tend to have lower rates of increased completeness between versions than those built using long reads (e.g. PacBio). FGMP_PROT and FGMP_HCE results are correlated (Spearman rho = 0.39) but clearly independent, which further highlights the utility of interrogating different genomic regions to assess completeness.

To further assess the ability of FGMP to detect missing genes and gene loss, we evaluated the impacts of randomly removing $\sim 10 \%$ of genomic sequence using 57 fungal genome assemblies (completeness estimates are provided in Additional file 4). FGMP_PROT successfully captures the degradations in all assemblies, the average loss rate was estimated at $5 \%$ instead of the original $10 \%$, which means the full extent of the simulated loss is not recovered. FGMP_HDE detected the loss of genomic regions in 54 assemblies with an average loss rate of $5.2 \%$. A search with BUSCO (fungi models) captures degradations in 55 assemblies with an average loss rate of $4.3 \%$. The removal of genomic regions prevented CEGMA pipeline from completing in many cases without apparent reasons. Therefore, we have concluded that CEGMA could only be run on the original genome assemblies. Examination of CEGMA completeness estimates found they are not statistically different from BUSCO (fungi models) estimations (Wilcoxon test, $p$-value 0.73), but differ significantly from FGMP results $(p$-value $=0.01$ ). These findings indicate FGMP and BUSCO perform relatively well on genome assemblies of varying degrees of completeness and are similar in estimations detecting degradations in genomes (Fig. 3). These conclusions hold when simulated loss of genomic regions ranged from 5 to 30\% (Additional file 4b).

\section{Genome completion and ecological or lifestyle traits}

We estimated the genome completeness of 166 fungal genomes (species details are described in Additional file 5). Only one version of the genome assembly was considered for each species. Each species was classified according to its lifestyle based on published literature (e.g. saprotroph, parasite; references are presented in Additional file 5). Parasites are typically characterized by a reduced genome size usually attributed their reliance, either partially or obligately, on nutrients scavenged from hosts. Their genomes are often enriched with transposable and repetitive elements, which in some extreme case composed more than $80 \%$ of the genomes [11]. Our dataset includes 34 pathogenic species which genome sizes range from $177.6 \mathrm{Mb}$ for the ectomycorrhizal fungus Cenococcum geophilum to $2.1 \mathrm{Mb}$ for the microsporidia Encephalitozoon romaleae. The remaining 132 fungi were classified as saprotopic and their genome sizes vary from $177 \mathrm{Mb}$ for the ectomycorrhizal fungus Cenococcum geophilum to $9.8 \mathrm{Mb}$ for the xerophilic fungus Wallemia sebi. Taking the whole set of genomes, the average $\mathrm{N} 50$ is $126.7 \mathrm{Mb}$ for an average number of scaffolds per genome of 1029; an average genome size of $38 \mathrm{Mb}$ and the average fraction of Ns per genome is $3.2 \%$.

Analysis of our set of 166 genome assemblies found that $92 \%$ have a CEGMA value $>95 \%$ whilst only $58.7 \%$ of these assemblies have a 95\% completeness with BUSCO fungi, 40\% with FGMP_HDE and 54.2\% with FGMP_PROT. Genomes labeled as incomplete are typically parasites, which suggest that gene losses from genome streamlining and missing sequence in assemblies might be confounded. These results indicate that a different set of representative sequence will be necessary for obligate parasites. Overall completeness predictions 


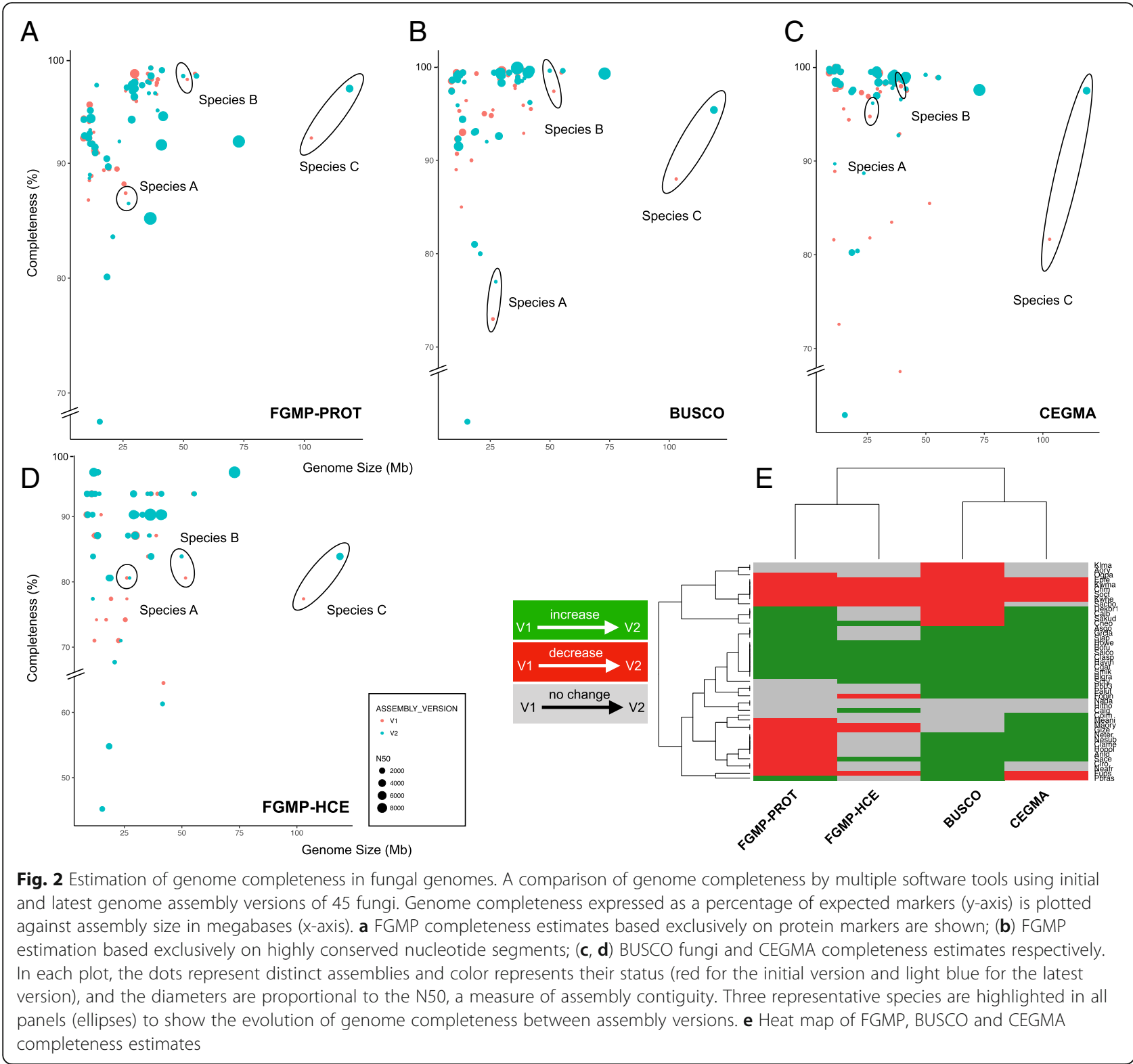

correlated with the N50: CEGMA (Spearman rho = $0.35, P$-value $\left.=1.3 \times 10^{-8}\right)$, BUSCO fungi $(\mathrm{R}=0.40 ; P=$ $\left.2.1 \times 10^{-11}\right)$, FGMP_HDE $(\mathrm{R}=0.17 ; \quad P=0.005)$ but FGMP_PROT $(\mathrm{R}=-0.05 ; P=0.4)$. FGMP_PROT predictions are not correlated with N50 as this metric incorporates gene fragments, which allow the partial detection of markers even when reliable gene models cannot be built. To avoid overcounting false positives and inflating the estimate, gene fragment sequences are required to score above a predetermined threshold to be accepted as valid hit. However, because short fragments are still required to display a significant similarity versus FGMP protein markers (scores), the likelihood of inflated completeness estimates is expected to be negligible.

\section{Runtimes}

We tracked the running times for 90 fungal genome assemblies (Additional file 6). Using six CPU cores each with $8 \mathrm{~GB}$ memory (AMD Opteron clock speed 2.1Ggz), FGMP runtimes are proportional to the size and the levels of fragmentation of the genomes under analysis. Runtimes are more influenced by the level of fragmentation of the assembly than its size (Additional file 7). For example, FGMP analysis is completed in $39 \mathrm{~min}$ for the 118 $\mathrm{Mb}$ genome size of $B$. graminis $(\mathrm{N} 50=2030.3 \mathrm{~kb})$ whereas the analysis of the $41 \mathrm{Mb}$ genome of Magnaporthe oryzae $(\mathrm{N} 50=153 \mathrm{~kb})$ requires three hours (Additional file 6). The fastest runtime observed was that of Cryptococcus gattii (assembly version 1 , size $17.1 \mathrm{Mb}, \mathrm{N} 50=44 \mathrm{~kb}$ ) completed 


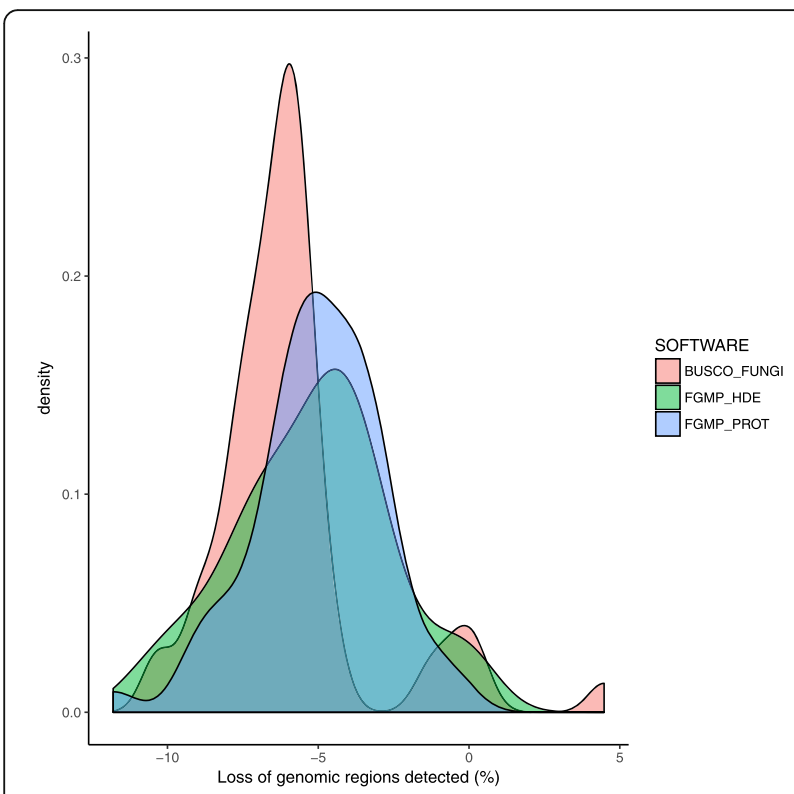

Fig. 3 Genomic loss simulations. The genomes of 57 fungal species were randomly truncated to evaluate accuracy of FGMP, BUSCO and CEGMA. The density plots represent the differences (expressed as percentages) between completeness estimates from the full and truncated assemblies

in $22 \mathrm{~min}$ and the longest with the genome of analysis of the $49.9 \mathrm{Mb}$ genome of Hortea werneckii (N50 $153 \mathrm{~kb}$ ) which required four hours.

\section{Discussion}

FGMP is a useful tool for automated assessment of genome assembly completeness of fungal genomes that incorporates measures of gene content covering both protein coding and noncoding regions. The tool combines multilevel analysis by scanning of both coding and non-coding regions of a given genome and provides a detailed reported describing the recovery of multiple types of genomic features in a genome assembly. Compared to existing methods BUSCO and CEGMA, FGMP fills a unique niche by assessing non-coding highly conserved segments and collapsed gene family's content in addition to measure of protein coding gene conservation. Additionally, FGMP does not rely exclusively on $a b$ inito gene predictions with tools like AUGUSTUS which require parameter training. FGMP reports complete, partial and aberrant gene models. FGMP also includes an experimental module, which allow a user to query raw reads using a reservoir sampling approach. This module is currently optimized for low input long reads similar to PacBio or Nanopore sequences. Future versions will include support for estimation from Illumina reads. FGMP has a modular architecture and thus can be easily incorporated into existing genome annotation pipelines.

\section{Conclusion}

A realistic estimation of level of genome completeness is a critical metric for accurate comparative genomics studies. This is particularly relevant as the sequencing costs decrease and whole genome assembly is attempted as daily routine for many purposes. BUSCO is currently the only maintained tool for such purpose. FGMP fills a unique niche in the sense that it has modules that assay additional feature types in genomes with no equivalent in existing methods. By applying FGMP to real and simulated datasets, we show that FGMP predictions are reliable and extended that of other software. The tool allows a deeper analysis in the context of evolutionary biology by quickly providing key metrics such the presence of potentially collapsed regions or can be used to screen reads before computationally costly genome assembly is attempted.

\section{Availability and requirements}

Project name: FGMP.

Project home page: https://github.com/stajichlab/FGMP Operating system(s): Linux, Mac OS.

Programming language: Perl 5.

License: MIT Open Source License.

Archived release: DOI: https://doi.org/10.5281/zenodo. 1453438

Package system availability: Bioconda.

Any restrictions to use by non-academics: none.

\section{Methods}

FGMP is written in Perl 5, and is designed for a command line interface. The code is organized into five distinct modules, which are stored in the main library "FGMP.pm".

1. Identify candidate regions: this module scans the genome assembly using 593 fungal proteins with TBLASTn [18]. In parallel, the assembly is translated using SIXPACK [19] and compared to 593 Hidden Markov models of the 593 protein markers using HMMER3 [20]. Long FASTA headers are discouraged.

2. Process alignments: aligns 593 protein makers to candidate regions using EXONERATE [22].

Alignments are converted in protein sequences and training sets for AUGUSTUS [23].

3. Annotation of candidate regions: uses AUGUSTUS to annotate the candidate regions. The module merges AUGUSTUS predictions with translated proteins from module 2 into a single FASTA file. The module compares raw predictions (proteins or peptides) to 593 HMMs using HMMSEARCH. Lastly, FGMP scans the original assembly for 31 universally conserved fungal elements using NHMMER. 
4. Check the status of multi-copy protein families: scans the raw predictions and identify markers that are expected to be in multiple copies. Markers with a lower number of copies than expected are tagged as potentially collapsed regions.

5. Generate final report: gather all raw predictions, filter aberrant predictions (at least twice the average length of the reference makers) and choose the longest gene model for each protein markers.

6. Infer genome completeness from long reads: is triggered when reads are provided. FGMP uses a reservoir sampling approach and BLASTx [18] to search for the 593 proteins markers in the reads.

\section{Additional files}

Additional file 1: List of fungal species used for phylogenomic analysis. (XLSX $42 \mathrm{~kb}$ )

Additional file 2: Phylogeny of nine fungal species used for the detection of highly conserved nucleotide elements. The divergence times were obtained from http://www.timetree.org [30] (PDF 25 kb)

Additional file 3: Comparison of protein markers used for genome completeness estimation. (PDF $231 \mathrm{~kb}$ )

Additional file 4: Assessment of genome completeness in 57 fungal genome assemblies. b Plots of genome completeness in 57 fungal genome assemblies. (ZIP $61 \mathrm{~kb}$ )

Additional file 5: Lifestyle, genome characteristics and completeness estimates of 166 fungi. (XLSX $47 \mathrm{~kb}$ )

Additional file 6: Genome characteristics, completeness estimates and run times of 90 fungal genomes. (XLSX $16 \mathrm{~kb}$ )

Additional file 7: Scatterplot showing the relationship between FGMP running times and the level of fragmentation for different genome assemblies expressed as N50. (PDF $5 \mathrm{~kb}$ )

\section{Abbreviations}

BLAST: Basic Local Alignment Search Tool; BUSCO: Benchmarking Universal Single-Copy Orthologs; CEGMA: Core Eukaryotic Genes Mapping Approach; EMBOSS: European Molecular Biology Open Software; FUNYBASE: FUNgal phylogenomic database; HDE: Highly conserved DNA elements; NCBI: National Center for Biotechnology Information; OMA: Orthologous Matrix

\section{Acknowledgements \\ Computations were performed on the University of California-Riverside Institute for Integrative Genome Biology high performance bioinformatics cluster (http:// hpcc.ucr.edu/) supported by NSF MRI DBI 1429826 and NIH S10-OD016290. This study used the Office of Cyber Infrastructure and Computational Biology (OCICB) high-performance computing (HPC) cluster at the National Institute of Al- lergy and Infectious Diseases (NIAID), Bethesda, MD.}

\section{Funding}

This work was supported by the Swiss National Science Foundation fellowship grant no. 151780 to O.H.C. NIH grant GM108492 and NSF grant DEB-1441715 to JES, partially supported these efforts. This work was supported by the USDA National Institute of Food and Agriculture Hatch project CA-R-PPA-5062-H. OHC is currently supported by an US National Institutes of Health Fogarty fellowship. This research was supported in part by the Intramural Research Program of the $\mathrm{NIH}$, Clinical Center. The funders had no role in the design of the study and collection, analysis, and interpretation of data and in writing the manuscript.

\section{Availability of data and materials}

The datasets generated during and/or analyzed during the current study are available at Zenodo (https://doi.org/10.5281/zenodo.2541782; https://doi.org/ 10.5281/zenodo.1453438).

\section{Author's contributions}

$\mathrm{OHC}$ and JES conceived of the project. OHC wrote the code. $\mathrm{OHC}$ and JES unit tested and wrote documentation. $\mathrm{OHC}$ generated the data utilized for testing. JES directed the project. OHC and JES wrote the manuscript. Both authors read and approved the final manuscript.

Ethics approval and consent to participate

Not applicable.

\section{Consent for publication}

Not applicable.

\section{Competing interests}

The authors declare that they have no competing interests.

\section{Publisher's Note}

Springer Nature remains neutral with regard to jurisdictional claims in published maps and institutional affiliations.

\section{Author details}

${ }^{1}$ Department of Microbiology \& Plant Pathology and Institute for Integrative Genome Biology, University of California-Riverside, Riverside, CA 92521, USA. ${ }^{2}$ Present address: Critical Care Medicine Department, NIH Clinical Center, National Institutes of Health, Bethesda, MD, USA.

Received: 22 October 2018 Accepted: 27 March 2019

Published online: 15 April 2019

\section{References}

1. Sohn JI, Nam JW. The present and future of de novo whole-genome assembly. Brief Bioinform. 2018;19:23-40.

2. Rinke C, Schwientek P, Sczyrba A, Ivanova NN, Anderson IJ, Cheng JF, Darling A, Malfatti S, Swan BK, Gies EA, Dodsworth JA, Hedlund BP, Tsiamis G, Sievert SM, Liu WT, Eisen JA, Hallam SJ, Kyrpides NC, Stepanauskas R, Rubin EM, Hugenholtz P, Woyke T. Insights into the phylogeny and coding potential of microbial dark matter. Nature. 2013;499:431-7.

3. Turnbaugh PJ, Ley RE, Hamady M, Fraser-Liggett CM, Knight R, Gordon J. The human microbiome project. Nature. 2007:449:804-10.

4. Spanu PD, Abbott JC, Amselem J, Burgis TA, Soanes DM, Stuber K, van Themaat EV, Brown JK, Butcher SA, Gurr SJ, Lebrun MH, Ridout CJ, SchulzeLefert P, Talbot NJ, Ahmadinejad N, Ametz C, Barton GR, Benjdia M, Bidzinski P, Bindschedler LV, Both M, Brewer MT, Cadle-Davidson L, Cadle-Davidson MM, Collemare J, Cramer R, Frenkel O, Godfrey D, Harriman J, Hoede C, King BC, Klages S, Kleemann J, Knoll D, Koti PS, Kreplak J, Lopez-Ruiz FJ, Lu X, Maekawa T, Mahanil S, Micali C, Milgroom MG, Montana G, Noir S, O'Connell RJ, Oberhaensli S, Parlange F, Pedersen C, Quesneville H, Reinhardt R, Rott M, Sacristan S, Schmidt SM, Schon M, Skamnioti P, Sommer H, Stephens A Takahara H, Thordal-Christensen H, Vigouroux M, Wessling R, Wicker T, Panstruga R. Genome expansion and gene loss in powdery mildew fungi reveal tradeoffs in extreme parasitism. Science. 2010;330:1543-6.

5. Kohler A, Kuo A, Nagy LG, Morin E, Barry KW, Buscot F, Canback B, Choi C, Cichocki N, Clum A, Colpaert J, Copeland A, Costa MD, Dore J, Floudas D, Gay G, Girlanda M, Henrissat B, Herrmann S, Hess J, Hogberg N, Johansson T, Khouja HR, LaButti K, Lahrmann U, Levasseur A, Lindquist EA, Lipzen A, Marmeisse R, Martino E, Murat C, Ngan CY, Nehls U, Plett JM, Pringle A, Ohm RA, Perotto S, Peter M, Riley R, Rineau F, Ruytinx J, Salamov A, Shah F, Sun H, Tarkka M, Tritt A, Veneault-Fourrey C, Zuccaro A, Mycorrhizal Genomics Initiative C, Tunlid A, Grigoriev IV, Hibbett DS, Martin F. Convergent losses of decay mechanisms and rapid turnover of symbiosis genes in mycorrhizal mutualists. Nat Genet. 2015;47:410-5.

6. Bradnam KR, Fass JN, Alexandrov A, Baranay P, Bechner M, Birol I, Boisvert S, Chapman JA, Chapuis G, Chikhi R, Chitsaz H, Chou WC, Corbeil J, Del Fabbro C, Docking TR, Durbin R, Earl D, Emrich S, Fedotov P, Fonseca NA, Ganapathy G, Gibbs RA, Gnerre S, Godzaridis E, Goldstein S, Haimel M, Hall G, Haussler D, Hiatt JB, Ho IY, Howard J, Hunt M, Jackman SD, Jaffe DB, Jarvis ED, Jiang H, Kazakov S, Kersey PJ, Kitzman JO, Knight JR, Koren S, Lam TW, Lavenier D, Laviolette F, Li Y, Li Z, Liu B, Liu Y, Luo R, Maccallum I, Macmanes MD, Maillet N, Melnikov S, Naquin D, Ning Z, Otto TD, Paten B, Paulo OS, Phillippy AM, Pina-Martins F, Place M, Przybylski D, Qin X, Qu C, Ribeiro FJ, Richards S, Rokhsar DS, Ruby JG, Scalabrin S, Schatz MC, Schwartz DC, Sergushichev A, Sharpe T, Shaw TI, Shendure J, Shi Y, Simpson JT, Song 
H, Tsarev F, Vezzi F, Vicedomini R, Vieira BM, Wang J, Worley KC, Yin S, Yiu SM, Yuan J, Zhang G, Zhang H, Zhou S, Korf IF. Assemblathon 2: evaluating de novo methods of genome assembly in three vertebrate species. Gigascience. 2013;2:10.

7. Parra G, Bradnam K, Korf I. CEGMA: a pipeline to accurately annotate core genes in eukaryotic genomes. Bioinformatics. 2007;23:1061-7.

8. Parra G, Bradnam K, Ning Z, Keane T, Korf I. Assessing the gene space in draft genomes. Nucleic Acids Res. 2009;37:289-97.

9. Waterhouse RM, Seppey M, Simao FA, Manni M, loannidis P, Klioutchnikov G, Kriventseva EV, Zdobnov EM. BUSCO applications from quality assessments to gene prediction and phylogenomics. Mol Biol Evol. 2018; 35(3):543-8.

10. Marthey S, Aguileta G, Rodolphe F, Gendrault A, Giraud T, Fournier E, LopezVillavicencio M, Gautier A, Lebrun MH, Chiapello H. FUNYBASE: a FUNgal phYlogenomic dataBASE. BMC Bioinformatics. 2008:9:456.

11. Stajich JE. Fungal Genomes and Insights into the Evolution of the Kingdom. Microbiol Spectr. 2016;5(4):FUNK-0055-2016. https://doi.org/10.1128/ microbiolspec.FUNK-0055-2016.

12. Salzberg SL, Phillippy AM, Zimin A, Puiu D, Magoc T, Koren S, Treangen TJ, Schatz MC, Delcher AL, Roberts M, Marcais G, Pop M, Yorke JA. GAGE: a critical evaluation of genome assemblies and assembly algorithms. Genome Res. 2012:22:557-67.

13. Yandell $M$, Ence $D$. A beginner's guide to eukaryotic genome annotation. Nat Rev Genet. 2012;13:329-42.

14. Hunt M, Kikuchi T, Sanders M, Newbold C, Berriman M, Otto TD. REAPR: a universal tool for genome assembly evaluation. Genome Biol. 2013:14:R47.

15. Gurevich A, Saveliev V, Vyahhi N, Tesler G. QUAST: quality assessment tool for genome assemblies. Bioinformatics. 2013;29:1072-5.

16. Gruning B, Dale R, Sjodin A, Chapman BA, Rowe J, Tomkins-Tinch CH, Valieris R, Koster J, Bioconda T. Bioconda: sustainable and comprehensive software distribution for the life sciences. Nat Methods. 2018;15:475-6.

17. Altenhoff AM, Skunca N, Glover N, Train CM, Sueki A, Pilizota I, Gori K, Tomiczek B, Muller S, Redestig H, Gonnet GH, Dessimoz C. The OMA orthology database in 2015: function predictions, better plant support, synteny view and other improvements. Nucleic Acids Res. 2015:43:D240-9.

18. Altschul SF, Madden TL, Schaffer AA, Zhang J, Zhang Z, Miller W, Lipman DJ, Gapped BLAST. PSI-BLAST: a new generation of protein database search programs. Nucleic Acids Res. 1997;25:3389-402.

19. Rice P, Longden I, Bleasby A. EMBOSS: the European molecular biology open software suite. Trends Genet. 2000;16:276-7.

20. Eddy SR. Accelerated profile HMM searches. PLoS Comput Biol. 2011; 7(10):e1002195.

21. Wallace IM, O'Sullivan O, Higgins DG, Notredame C. M-Coffee: combining multiple sequence alignment methods with T-Coffee. Nucleic Acids Res. 2006;34:1692-9.

22. Slater GS, Birney E. Automated generation of heuristics for biological sequence comparison. BMC bioinformatics. 2005;6:31.

23. Stanke M, Schoffmann O, Morgenstern B, Waack S. Gene prediction in eukaryotes with a generalized hidden Markov model that uses hints from external sources. BMC bioinformatics. 2006;7:62.

24. Jones P, Binns D, Chang HY, Fraser M, Li W, McAnulla C, McWilliam H, Maslen J, Mitchell A, Nuka G, Pesseat S, Quinn AF, Sangrador-Vegas A, Scheremetjew M, Yong SY, Lopez R, Hunter S. InterProScan 5: genome-scale protein function classification. Bioinformatics. 2014;30:1236-40.

25. Kielbasa SM, Wan R, Sato K, Horton P, Frith MC. Adaptive seeds tame genomic sequence comparison. Genome Res. 2011;21:487-93.

26. Quinlan AR, Hall IM. BEDTools: a flexible suite of utilities for comparing genomic features. Bioinformatics. 2010;26:841-2.

27. Bailey TL, Boden M, Buske FA, Frith M, Grant CE, Clementi L, Ren J, Li WW, Noble WS. MEME SUITE: tools for motif discovery and searching. Nucleic Acids Res. 2009:37:W202-8

28. Kalvari I, Nawrocki EP, Argasinska J, Quinones-Olvera N, Finn RD, Bateman A, Petrov Al. Non-coding RNA analysis using the Rfam database. Curr Protoc Bioinformatics. 2018:62:e51.

29. Lopez-Ramirez V, Alcaraz LD, Moreno-Hagelsieb G, Olmedo-Alvarez G. Phylogenetic distribution and evolutionary history of bacterial DEAD-box proteins. J Mol Evol. 2011;72:413-31.

30. Kumar S, Stecher G, Suleski M, Hedges SB. TimeTree: a resource for timelines, Timetrees, and divergence times. Mol Biol Evol. 2017;34:1812-9.

Ready to submit your research? Choose BMC and benefit from:

- fast, convenient online submission

- thorough peer review by experienced researchers in your field

- rapid publication on acceptance

- support for research data, including large and complex data types

- gold Open Access which fosters wider collaboration and increased citations

- maximum visibility for your research: over $100 \mathrm{M}$ website views per year

At $\mathrm{BMC}$, research is always in progress.

Learn more biomedcentral.com/submissions 